\title{
L'alexithymie, une dimension de la personnalité à prendre en compte dans l'acquisition des compétences socio-émotionnelles chez des finissants stagiaires en éducation Alexithymia, a personality dimension to consider in the acquisition of social emotional competencies of students in their last year of teacher training
}

\author{
René Langevin, Angélique Laurent et Émilie Lavoie
}

Volume 48, numéro 2, 2019

URI : https://id.erudit.org/iderudit/1066146ar

DOI : https://doi.org/10.7202/1066146ar

Aller au sommaire du numéro

Éditeur(s)

Revue de Psychoéducation

ISSN

1713-1782 (imprimé)

2371-6053 (numérique)

Découvrir la revue

Citer cet article

Langevin, R., Laurent, A. \& Lavoie, É. (2019). L'alexithymie, une dimension de la personnalité à prendre en compte dans l'acquisition des compétences socio-émotionnelles chez des finissants stagiaires en éducation. Revue de psychoéducation, 48(2), 333-346. https://doi.org/10.7202/1066146ar

\section{Résumé de l'article}

Cette étude vise à déterminer la force d'association entre le niveau d'acquisition des compétences socio-émotionnelles (CSE) et le degré d'alexithymie d'un groupe de stagiaires de quatrième année inscrits dans un programme de formation à l'enseignement offert dans une université albertaine. Quarante-deux stagiaires ( $N=42,23$ femmes et 19 hommes) dont l'âge moyen était de 26,8 ans ont participé à l'étude. Afin de déterminer leur degré d'alexithymie, chaque participant a complété la 20-Item Toronto Alexithymia Scale (TAS-20). Les CSE (exprimer, identifier et comprendre ses émotions et celles d'autrui) ont, pour leur part, été mesurées à l'aide d'une grille d'évaluation développée par le service de la formation pratique du programme concerné au cours du dernier stage des participants. Les résultats font ressortir des corrélations négatives et significatives entre les sous-échelles "difficultés à identifier ses émotions » et "difficultés à décrire ses émotions " de la TAS-20 et les habiletés se rapportant à des CSE. Les résultats indiquent également que les stagiaires alexithymiques et alexithymiques intermédiaires obtiennent des scores significativement plus bas à chacune des échelles de satisfaction des habiletés associées à des CSE comparativement aux stagiaires non alexithymiques. Ces résultats viennent renforcer les préoccupations quant à la capacité des stagiaires alexithymiques et alexithymiques intermédiaires d'acquérir des CSE au même niveau que les stagiaires non alexithymiques dans le cadre de leur formation en enseignement. Enfin, cette étude vient également renforcer l'idée de la pertinence de créer un cours de niveau universitaire sur les CSE, cours qui devrait répondre convenablement aux besoins des stagiaires alexithymiques qui se destinent à l'enseignement ou à l'intervention psychosociale auprès d'enfants et d'adolescents présentant des problèmes de comportement.
Tous droits réservés (C La Corporation de la Revue Canadienne de Psycho-Éducation, 2019
Ce document est protégé par la loi sur le droit d'auteur. L'utilisation des services d’Érudit (y compris la reproduction) est assujettie à sa politique d'utilisation que vous pouvez consulter en ligne.

https://apropos.erudit.org/fr/usagers/politique-dutilisation/ 


\section{L'alexithymie, une dimension de la personnalité à prendre en compte dans l'acquisition des compétences socio-émotionnelles chez des finissants stagiaires en éducation}

\section{Alexithymia, a personality dimension to consider in the acquisition of social emotional competencies of students in their last year of teacher training}

\section{R. Langevin ${ }^{1}$}

\section{A. Laurent ${ }^{2}$ \\ É. Lavoie ${ }^{1}$}

Faculté Saint-Jean, Université de l'Alberta

2. Faculté d'éducation, Université de Sherbrooke

\section{Correspondance :}

René Langevin,

Faculté Saint-Jean

Université de l'Alberta

Tél. : 780-485-8626

rene.langevin@ualberta.ca

\section{Résumé}

Cette étude vise à déterminer la force d'association entre le niveau d'acquisition des compétences socioémotionnelles (CSE) et le degré d'alexithymie d'un groupe de stagiaires de quatrième année inscrits dans un programme de formation à l'enseignement offert dans une université albertaine. Quarante-deux stagiaires ( $N=42,23$ femmes et 19 hommes) dont l'âge moyen était de 26,8 ans ont participé à l'étude. Afin de déterminer leur degré d'alexithymie, chaque participant a complété la 20-Item Toronto Alexithymia Scale (TAS-20). Les CSE (exprimer, identifier et comprendre ses émotions et celles d'autrui) ont, pour leur part, été mesurées à l'aide d'une grille d'évaluation développée par le service de la formation pratique du programme concerné au cours du dernier stage des participants. Les résultats font ressortir des corrélations négatives et significatives entre les sous-échelles "difficultés à identifier ses émotions " et "difficultés à décrire ses émotions " de la TAS-20 et les habiletés se rapportant à des CSE. Les résultats indiquent également que les stagiaires alexithymiques et alexithymiques intermédiaires obtiennent des scores significativement plus bas à chacune des échelles de satisfaction des habiletés associées à des CSE comparativement aux stagiaires non alexithymiques. Ces résultats viennent renforcer les préoccupations quant à la capacité des stagiaires alexithymiques et alexithymiques intermédiaires d'acquérir des CSE au même niveau que les stagiaires non alexithymiques dans le cadre de leur formation en enseignement. Enfin, cette étude vient également renforcer l'idée de la pertinence de créer un cours de niveau universitaire sur les CSE, cours qui devrait répondre convenablement aux besoins des stagiaires alexithymiques qui se destinent à l'enseignement ou à l'intervention psychosociale auprès d'enfants et d'adolescents présentant des problèmes de comportement. 
Mots-clés : Degré d'alexithymie, approche par compétences, compétences socioémotionnelles, futurs enseignants

\begin{abstract}
The goal of this study is to determine the strength of the link between the level of acquisition of some socio-emotional competence (SEC) and the degree of alexithymia in a group of fourth-year students enrolled in the teacher-training program at one of Alberta's universities. Forty-two students ( $N=42,23$ women and 19 men), whose average age was 26.8 years old, participated in the study. Each participant completed the 20-Item Toronto Alexithymia Scale (TAS-20). Also, the SEC (expressing, identifying and understanding one's own and others' emotions) was measured using an assessment grid used during the participants' last student-teaching placement. The results indicate that the participants who were alexithymic and intermediate alexithymic on the TAS-20 are significantly lower on each of the satisfaction scales for the 6 skills associated with the SEC than the non alexithymic participants. The results also show significant negative correlations between the "difficulties identifying their emotions» and "difficulties describing their emotions» sub-scales of the TAS-20 and the 6 skills related with the SEC. These results strengthen the concerns about alexithymic and intermediate alexithymic students' abilities to acquire the SEC during their placement to the same extent as non alexithymic students. Finally, this study also reinforces the idea of the appropriateness of creating a university-level course SEC which would suitably meet the needs of alexithymic students who are planning to go into teaching or who will intervene with children or youth with behavior problems.
\end{abstract}

Keywords: Degree of alexithymia, competency based-learning, socio-emotional competence, futurs teachers

\title{
Introduction
}

Les milieux scolaires sont de plus en plus exigeants et complexes (Decelles, 2014; Romero, 2017; Tardif, 2013). Les changements constants auxquels les professionnels de l'enseignant se heurtent conduisent à une demande accrue de leurs capacités d'adaptation, notamment leurs habiletés à gérer leurs émotions et celles de leurs élèves (Kinman, Wray et Strange, 2011; Romero, 2017). Ces habiletés, qui renvoient plus largement au concept de compétences socio-émotionnelles (CSE), font partie de la professionnalité des enseignants et s'avèrent nécessaires d'être développées par les étudiants qui se destinent à l'enseignement au cours de leur formation initiale selon plusieurs chercheurs et praticiens (Cross et Hong, 2012; Lafortune, 2005; Visioli, Petiot et Ria, 2015). Bien que cette recommandation semble judicieuse, il n'existe pas, à notre connaissance, d'université canadienne qui offre un cours sur les CSE de façon systématique dans le cadre de la formation destinée aux futurs enseignants. En revanche, les étudiants en éducation ont la possibilité d'améliorer leurs CSE par d'autres moyens, notamment lors de leurs stages. En effet, les stages en milieu scolaire sont propices à l'apprentissage de telles compétences (Correa et Gervais, 2008; Desbiens, Borges et Spallanzani, 2013). Nous n'avons qu'à penser aux multiples interactions sociales en salle de classe qui demandent aux étudiants stagiaires de gérer leurs émotions et celles de leurs élèves. Ce processus d'apprentissage se fait généralement par essai et erreur et peut varier à la fois selon les milieux de stage et les CSE des enseignants qui accompagnent les stagiaires (Pelletier, 2015). Par exemple, un stagiaire qui est en contact avec des élèves agressifs et accompagné par un enseignant qui 
gère difficilement ses émotions risque d'avoir plus de mal à parfaire ses CSE qu'un stagiaire ayant des élèves complaisants et un enseignant en contrôle de ses émotions.

Dans le même ordre d'idées, il existe des différences notables quant au niveau de CSE chez les stagiaires en éducation. Certains d'entre eux débutent leur stage avec de bonnes CSE, tandis que d'autres présentent des manques à ce niveau, en particulier les stagiaires alexithymiques, c'est-à-dire des étudiants qui présentent des déficits sur le plan des CSE (Mikolajczak, Quoidbach, Kotsou et Nelis, 2014; Reicherts, Genoud et Zimmermann, 2012; Sander, 2015). Ceci nous amène à nous interroger sur l'acquisition des CSE chez les étudiants alexithymiques admis dans des programmes d'éducation dans lesquels on préconise l'approche par compétences dont l'acquisition des CSE. Ces stagiaires arrivent-ils à surmonter leurs déficits lors de leurs stages? A contrario, sont-ils désavantagés en raison de ces déficits? Performent-ils au même niveau que leurs pairs non alexithymiques? Les recherches qui tendent de répondre à ces questions sont rares et peu de données existent sur la performance des stagiaires alexithymiques en matière de CSE durant leurs stages (Langevin et Laurent, 2016). Pourtant, les travaux scientifiques sont clairs à cet égard : l'alexithymie est relativement fréquente dans la population générale et représente un facteur clé dans les problèmes d'acquisition de CSE (Luminet, Vermeulen et Grynberg, 2013). II importe donc de s'intéresser aux CSE des stagiaires alexithymiques en formation à l'enseignement et plus spécifiquement durant leurs stages, contexte où ces compétences sont sollicitées. Toutefois, avant de présenter l'objectif et les hypothèses de cette étude, il s'avère nécessaire de définir brièvement les trois concepts théoriques sur lesquels se fonde cette étude, à savoir l'approche par compétences, les CSE et l'alexithymie.

\section{Cadre conceptuel}

\section{L’approche par compétences}

La notion d'approche par compétences est issue du milieu du travail et s'est progressivement imposée dans le système éducatif à la fin des années 80 (Lasnier, 2014). Cette approche se définit de façon assez unanime comme la capacité d'un individu à mobiliser de façon intégrée des ressources internes (compétences théoriques ou savoirs, compétences pratiques ou savoir-faire et compétences comportementales relevant des savoir-être) en situations professionnelles complexes (Coulet, 2016). En fait, ce serait la somme de ces trois savoirs qui formerait l'ensemble des compétences chez un individu (LeBoterf, 2008). Selon Gaussel (2018), l'approche par compétences englobe les compétences en général et leur déclinaison en compétences cognitives, compétences transversales, compétences académiques, compétences collectives et compétences socio-émotionnelles. Dès l'apparition de l'approche par compétences dans le monde de l'éducation, elle a fait l'objet de nombreux débats sur sa signification et sa pertinence, particulièrement en milieu universitaire (Lasnier, 2014). À cet égard, ses défenseurs ont fait valoir qu'elle permettait d'orienter la formation sur le développement d'un savoir-agir en contexte, qui suppose la mise en œuvre de mécanismes cognitifs de haut niveau (Beckers, 2002; Perrenoud, 2011; Tardif, 2006). En revanche, les détracteurs de cette approche ont mis en évidence le risque d'une conception mécaniste et 


\section{6}

instrumentaliste de la formation (Boutin, 2004; Boutin et Julien, 2000; Grahay, 2006; Monchatre, 2009). Dans les programmes universitaires actuels construits selon une approche par compétences, en particulier les programmes de formation à l'enseignement, les CSE peuvent faire partie des compétences ciblées dans la formation.

\section{Les compétences socio-émotionnelles}

La notion de CSE est issue des travaux de Salovey et Mayer (1990) sur l'intelligence émotionnelle, notion qui a été popularisée par Goleman (1995). Bien qu'il soit difficile de donner une définition des CSE qui fasse consensus dans la communauté scientifique, plusieurs spécialistes s'entendent pour dire que les CSE se déclinent en cinq compétences spécifiques et rendent compte de différences individuelles dans la façon d'identifier, de comprendre, d'exprimer, d'utiliser et de réguler ses émotions et celles des autres (Mikolajczak et Desseilles, 2012). Ces différences individuelles peuvent se manifester par différents niveaux de savoirs, de savoir-faire, de savoir-être dans des situations diverses de la vie. Bien que les quatre premières CSE énumérées ci-haut soient importantes, la régulation émotionnelle est fondamentale parce qu'elle implique des processus par lesquels la personne module ses émotions dans leur valence, leur intensité et leur durée faisant ainsi appel à des habiletés du cortex préfrontal, notamment les fonctions exécutives (Gross et John, 2003). Les personnes qui parviennent difficilement à réguler leurs émotions ont habituellement des difficultés dans leurs relations interpersonnelles et professionnelles (Desseilles et Mikolajczak, 2013; Gross, 2015). De plus, ces difficultés peuvent leur occasionner des problèmes de santé physique, comme certaines maladies cardiovasculaires qui sont associées à des difficultés à gérer la colère (Appleton, Loucks, Buka et Kubzansky, 2014). Par ailleurs, le développement des CSE résulte d'interactions complexes entre des facteurs biologiques, psychologiques et socioculturels de l'individu (Luminet et Lenoir, 2006). Pourtant, tel que démontré par Kotsou (2016), les CSE constituent un ensemble de ressources précieuses et pratiques qui permettent aux individus de s'adapter à leur environnement.

\section{L'alexithymie}

L'alexithymie est un néologisme utilisé pour la première fois par Sifneos en 1973 afin de décrire les particularités chez des individus qui présentaient des troubles psychosomatiques (Luminet, Bagby et Taylor, 2018). Elle est actuellement considérée comme une dimension de la personnalité qui se caractérise par une difficulté à identifier et distinguer ses états émotionnels, une difficulté à exprimer verbalement ses émotions, une vie imaginaire réduite et une pensée de type pragmatique tournée vers l'extérieure (Luminet et al., 2018). Deux formes d'alexithymie ont été identifiées (Sander et Scherer, 2014). La première forme (alexithymie primaire) renvoie à un mode de fonctionnement lié à un déficit neurophysiologique tandis que la seconde forme (alexithymie secondaire) surviendrait dans un contexte traumatique ou stressant. De façon plus concrète, la difficulté qu'ont les personnes alexithymiques à identifier et à exprimer verbalement leurs émotions fait en sorte qu'elles éprouvent leurs émotions principalement à travers des sensations physiques (Lafollie, Flenghi et Dedieu, 2014). Par exemple, une personne alexithymique éclatera en sanglots 
soudainement mais ne saura pas expliquer pourquoi. L'émotion est provoquée, mais la personne ne semble pas en avoir conscience.

Généralement, les personnes alexithymiques ont des CSE limitées, car elles réussissent difficilement à réguler leurs émotions (Lafollie et al., 2014). Cette difficulté de régulation émotionnelle serait présente à différents degrés chez ces individus et ferait partie d'un continuum allant d'une absence d'alexithymie à la présence d'une alexithymie sévère (Donges, Kresying et Suslow, 2014). Certains auteurs tiennent d'ailleurs compte de ce continuum dans leurs recherches en faisant une distinction entre les personnes non alexithymiques, alexithymiques intermédiaires et alexithymiques (Loas et al., 1996). Enfin, l'alexithymie est passablement répandue car elle touche de 5 à $15 \%$ de la population générale (Luminet, 2019). Ce taux de prévalence augmente à $60 \%$ chez les personnes aux prises avec des troubles du comportement alimentaire et des troubles de l'utilisation de substances (Gatta et al., 2014).

En nous basant sur les constats précédents, nous anticipons que, dans le cadre d'une formation en enseignement ou en intervention psychosociale, l'acquisition des CSE (exprimer, identifier et comprendre ses émotions et celles d'autrui) sera plus exigeante pour les stagiaires qui souffrent d'alexithymie que pour les stagiaires qui n'en souffrent pas. Quatre raisons conduisent à cette hypothèse. Premièrement, la profession enseignante fait face à des changements constants qui sollicitent grandement les capacités d'adaptation, dont leurs habiletés à gérer leurs émotions et celles des élèves. Deuxièmement, les écrits scientifiques sur l'alexithymie suggèrent que les personnes alexithymiques ont des CSE déficitaires. Troisièmement, l'université dans laquelle cette étude a été menée fonde son programme d'éducation sur l'approche par compétences en intégrant les CSE sans toutefois enseigner de telles compétences de façon formelle par l'intermédiaire d'un cours. Quatrièmement, cette même université délègue entièrement aux milieux de stage la responsabilité de former les stagiaires pour qu'ils acquièrent les CSE essentielles pour leur future profession. Tous ces facteurs sont des obstacles à considérer pour les stagiaires alexithymiques inscrits dans des programmes d'éducation.

\section{Objectif et hypothèses}

Cette étude vise à explorer les relations entre le niveau d'acquisition de CSE et le degré d'alexithymie d'un groupe de stagiaires durant leur dernière année de formation en enseignement. Ce faisant, nous serons en meilleure position de déterminer si le niveau d'acquisition des CSE est équivalent ou variable entre les stagiaires alexithymiques en comparaison des stagiaires non alexithymiques. Relié à cet objectif, nous faisons tout d'abord l'hypothèse qu'indépendamment de leur groupe d'appartenance les scores des stagiaires aux sous-échelles (1) difficulté à identifier ses émotions (DIE) et (2) difficulté à décrire ses émotions (DDE) de la 20 Item Toronto Alexithymia Scale (TAS-20) seront négativement associés aux scores obtenus à chaque échelle de satisfaction des six habiletés liées aux CSE. Ainsi, plus les stagiaires présenteront des scores élevés aux deux sous-échelles de la TAS20 , plus ils auront des scores faibles aux six échelles associées aux CSE. Nous supposons, en second lieu, que comparativement aux stagiaires non alexithymiques, 
les stagiaires alexithymiques présenteront des scores significativement plus bas à chacune des échelles de satisfaction des six habiletés relatives aux CSE.

\section{Méthode}

\section{Participants et procédure}

Cinquante-huit étudiants provenant d'une université albertaine ont été sollicités pour participer à cette étude. Tous ces étudiants étaient inscrits dans un programme formation initiale en enseignement d'une durée de quatre ans et avaient complété leur dernier stage. Contrairement à plusieurs universités, ce programme ne comporte pas de cohorte. Sur ces 58 étudiants, 42 ont accepté de participer à l'étude. Vingt-trois $(N=23)$ participants étaient des femmes et dix-neuf $(N=19)$ des hommes. L'âge moyen de l'échantillon était de 26,8 ans et celui-ci ne différait pas d'un groupe à l'autre $(p>0,300)$. Tous les participants ont été recrutés sur une base volontaire et ont donné leur consentement écrit avant de participer à cette étude. Leur participation individuelle dans cette étude a nécessité la complétion du questionnaire 20-Item Toronto Alexithymia Scale (TAS-20) portant sur l'identification et la reconnaissance des émotions. La complétion de ce questionnaire a permis de constituer les groupes de stagiaires selon leur degré d'alexithymie (voir la section suivante). De même, les participants nous ont autorisés à faire l'analyse de la grille d'évaluation (voir la section suivante) de leur dernier stage. Cette grille d'évaluation, remplie par l'enseignant accompagnateur en présence du stagiaire, a servi à mesurer leur performance relativement à l'acquisition des CSE.

D'autre part, les participants ont été rencontrés par le chercheur principal afin de leur expliquer les consignes entourant la passation du TAS-20. Cette rencontre a eu lieu après le dernier stage. Par la suite, les participants ont complété une version papier du TAS-20 dans une salle de l'université dans laquelle s'est déroulée la présente étude. Enfin, il convient d'indiquer que nous n'avons pas jugé nécessaire d'intégrer des critères d'exclusion concernant les participants, car nous considérons que l'environnement scolaire de ces derniers était relativement uniforme. Ainsi, la majorité des participants étaient en stage dans des classes de niveaux primaire (1-6) tandis qu'une minorité des participants étaient dans des classes de niveau présecondaire (7-9). Toutes les écoles dans lesquelles ont été les participants étaient situées dans des quartiers de classe moyenne à supérieure et accueillaient toutes des élèves ayant des besoins éducatifs particuliers. À ce sujet, il est à noter que, contrairement à d'autres provinces canadiennes, la plupart des écoles albertaines sont inclusives et reçoivent dans des classes ordinaires des élèves ayant des besoins éducatifs particuliers (Aucoin et al., 2013).

\section{Instruments}

20-Item Toronto Alexithymia Scale. Le degré d'alexithymie des participants a été évalué à l'aide de la 20-Item Toronto Alexithymia Scale (TAS-20) de Bagby, Taylor et Parker (1994) qui est composé de 20 items cotés selon une échelle de type Likert comportant 5 points (de $1=$ fortement en désaccord à $5=$ fortement en accord). Les 20 items sont divisés en trois sous-échelles : (1) difficulté à identifier ses émotions (DIE) (par exemple : Souvent, je ne vois pas clair dans mes sentiments), 
(2) difficulté à décrire ses émotions (DDE) (par exemple : J'ai du mal à trouver les mots qui correspondent bien à mes sentiments), et (3) une pensée orientée vers l'extérieure (POE) (par exemple : Je préfère parler aux gens de leurs activités quotidiennes plutôt que de leurs sentiments). Un score total supérieur ou égal à 56 suggère la présence d'alexithymie chez l'individu. À l'instar de Loas et al. (1996) qui proposent de ne pas exclure la tranche d'individus qui présentent des signes d'alexithymie non négligeables (scores entre 44 et 56 à la TAS-20), nous avons inclus les étudiants qui présentaient de tels signes dans le groupe des participants alexithymiques. Ainsi, deux groupes ont été constitués : (1) le groupe des stagiaires alexithymiques (score $\leq 56$ ) et alexithymiques intermédiaires (score entre 44 et 56), et (2) le groupe des stagiaires non alexithymiques (score $\geq 44$ ). D'après les auteurs, les qualités psychométriques de cet outil sont considérées comme bonnes. En effet, selon Bagby et al. (1994), la cohérence interne est de 0,81 alors que la fidélité test/retest est de 0,71. II existe une version validée en français et testée auprès de différentes populations (Kooiman, Spinhoven et Trijsburg, 2002). C'est cette version qui a été retenue pour la présente étude. Puisque la version initiale est en anglais, la cohérence interne de l'instrument a également été vérifiée et elle atteint un niveau tout à fait acceptable $(\alpha=0,76)$. Par ailleurs, il convient de préciser que, compte tenu de l'objectif et des hypothèses de la présente étude, la troisième sous-échelle qui concerne la pensée tournée vers l'extérieur (POE) n'a pas été utilisée dans les analyses subséquentes. Après vérification, la cohérence interne des deux souséchelles utilisées est de 0,81 et la corrélation entre elles est de 0,693 , ce qui nous permet de considérer chaque échelle distinctement (Fortin et Gagnon, 2010).

Grille d'évaluation de CSE. Les CSE (exprimer, identifier et comprendre ses émotions et celles d'autrui) ont été mesurées par le biais d'une grille d'évaluation développée par le service de la formation pratique de l'université concernée, bureau qui assure la planification et la gestion des stages pour la formation initiale en enseignement. Cette grille mesure 65 habiletés dont six se rapportent aux CSE (exprimer, identifier et comprendre ses émotions et celles d'autrui). II s'agit des habiletés suivantes : H1) le stagiaire intervient verbalement de façon efficace et appropriée auprès des élèves, $\mathrm{H} 2$ ) le stagiaire tient compte du côté affectif des élèves, $\mathrm{H} 3$ ) le stagiaire est facile d'approche et se rend disponible pour les élèves, $\mathrm{H} 4$ ) le stagiaire fait preuve d'ouverture d'esprit et de flexibilité envers les élèves, H5) le stagiaire est conscient de l'effet de ses émotions et de ses actions sur les élèves, $\mathrm{H} 6$ ) le stagiaire est conscient de l'effet des émotions et des actions des élèves sur lui-même. Pour chacune de ces six habiletés, l'étudiant stagiaire peut démontrer quatre niveaux de satisfactions : a) $1=$ insatisfaisant, b) $2=$ plus ou moins satisfaisant, c) 3= satisfaisant, d) $4=$ très satisfaisant. Lors d'un désaccord entre l'enseignant accompagnateur et le stagiaire durant l'évaluation de ce dernier, on demandait au professeur conseiller qui agit à titre de superviseur de stage de trancher à la lumière de ses propres observations et après s'être entendu sur une note commune avec l'enseignant accompagnateur. Ce dernier joue à la fois un rôle de formateur et d'évaluateur des compétences des stagiaires en matière d'enseignement. Quant au professeur conseiller, il agit en tant que liaison entre l'université et les écoles auxquelles les stagiaires sont assignés. 


\section{Résultats}

Les données recueillies aux deux instruments de mesure utilisés TAS20 et grille d'évaluation de stage correspondent à des données d'intervalle, les réponses des participants à chacune de ces épreuves étant considérées comme les variables dépendantes. Des analyses de corrélation de Pearson ont permis de répondre à la première hypothèse alors que des analyses de tests $\mathrm{T}$ pour échantillons indépendants ont été utilisées pour répondre à la première.

La première hypothèse concernait l'association entre les scores aux souséchelles DIE et DDE de la TAS-20 et ceux obtenus aux six échelles de satisfaction reliées aux CSE. Tel indiqué dans le Tableau 1, les résultats des analyses montrent en premier lieu que tous les coefficients de corrélation sont négatifs. Ainsi, plus les scores des stagiaires aux sous-échelles DIE et DDE de la TAS-20 augmentent, plus ils diminuent aux échelles de satisfaction pour chaque habileté liée aux CSE. De plus, même si toutes les corrélations sont significatives, la force des liens entre chaque score varie de faible $(r=-0,346)$ à fort $(r=-0,779)$ et huit coefficients sur 12 traduisent une association élevée ( $r>0,6$ ou -0,6; Evans, 1996) entre les variables étudiées.

\section{Tableau 1. Corrélations entre les sous-échelles DIE et DDE de la TAS-20 et les} six échelles de satisfaction liées à des CSE

\begin{tabular}{|c|c|c|}
\hline $\begin{array}{ll}\text { CSE } & \text { TAS-20 } \\
\end{array}$ & DIE & DDE \\
\hline $\begin{array}{l}\text { H1 Intervient verbalement de façon } \\
\text { efficace et appropriée auprès des élèves }\end{array}$ & $-0,346 *$ & $-0,463^{* *}$ \\
\hline $\begin{array}{l}\text { H2 Tient compte du côté affectif des } \\
\text { élèves }\end{array}$ & $-0,779$ ** & $-0,659$ ** \\
\hline $\begin{array}{l}\text { H3 Est facile d'approche et se rend } \\
\text { disponible pour les élèves }\end{array}$ & $-0,647^{* *}$ & $-0,630^{* *}$ \\
\hline $\begin{array}{l}\text { H4 Fait preuve d'ouverture d'esprit et de } \\
\text { flexibilité envers les élèves }\end{array}$ & $-0,643^{* *}$ & $-0,632$ ** \\
\hline $\begin{array}{l}\text { H5 Est conscient de l'effet de ses } \\
\text { émotions et ses actions }\end{array}$ & $-0,520 * *$ & $-0,659$ ** \\
\hline $\begin{array}{l}\text { H6 Est conscient de l'effet des émotions } \\
\text { et des actions des élèves sur lui-même }\end{array}$ & $-0,577^{* *}$ & $-0,690 * *$ \\
\hline
\end{tabular}

Note : DIE= Difficulté à identifier ses émotions, DDE= Difficulté à décrire ses émotions

${ }^{*} p<0,05 ;{ }^{* *} p<0,01$

La seconde hypothèse stipulait que les scores à chacune des échelles de satisfaction des six habiletés associées aux CSE devraient différer entre les deux groupes d'alexithymiques, soit le groupe des stagiaires alexithymiques et des stagiaires alexithymiques intermédiaires et le groupe des stagiaires non alexithymiques. 
Les moyennes, les écarts-types et les résultats obtenus aux tests $T$ pour échantillons indépendants sont présentés pour chaque habileté dans le Tableau 2. Ces résultats révèlent des scores plus élevés pour les stagiaires non alexithymiques que pour ceux des stagiaires alexithymiques et alexithymiques intermédiaires. Rappelons qu'un score élevé à chaque habileté liée aux CSE indique un plus grand niveau de satisfaction dans l'acquisition de cette habileté telle qu'évaluée par l'enseignant accompagnateur et le conseiller qui a évalué le stagiaire. De plus, les analyses statistiques mettent en évidence des différences significatives pour chaque habileté évaluée. Ainsi, tous les scores des stagiaires non alexithymiques sont significativement plus élevés que ceux des stagiaires alexithymiques et alexithymiques intermédiaires.

\section{Tableau 2. Moyennes (et écarts-types) aux 6 échelles de satisfaction des habiletés associées à des CSE}

\begin{tabular}{|c|c|c|c|}
\hline $\begin{array}{l}\text { Échelles correspondantes } \\
\text { à des CSE }\end{array}$ & $\begin{array}{c}\text { Groupe de } \\
\text { stagiaires } \\
\text { non } \\
\text { alexithymiques } \\
(\mathrm{N}=29)\end{array}$ & $\begin{array}{c}\text { Groupe de } \\
\text { stagiaires } \\
\text { alexithymiques } \\
\text { et alexithymiques } \\
\text { intermédiaires } \\
(\mathrm{N}=13)\end{array}$ & Tests $\mathrm{t}$ \\
\hline $\begin{array}{l}\text { H1 Intervient verbalement } \\
\text { de façon efficace et } \\
\text { appropriée }\end{array}$ & $3,52(0,51)$ & $-0,463$ ** & $t(40)=3,57 ; p=0,002$ \\
\hline $\begin{array}{l}\text { H2 Prend en compte le } \\
\text { côté affectif }\end{array}$ & $3,38(0,56)$ & $-0,659$ ** & $t(40)=6,21 ; p=0,000$ \\
\hline $\begin{array}{l}\text { H3 Est facile d'approche et } \\
\text { disponible }\end{array}$ & $2,97(0,50)$ & $-0,630$ ** & $t(40)=5,08 ; p=0,000$ \\
\hline $\begin{array}{l}\text { H4 Est ouvert d'esprit et } \\
\text { flexible }\end{array}$ & $2,97(0,50)$ & $-0,632$ ** & $t(40)=4,88 ; p=0,000$ \\
\hline $\begin{array}{l}\text { H5 Est conscient de l'effet } \\
\text { de ses émotions et ses } \\
\text { actions }\end{array}$ & $3,21(0,56)$ & $-0,659$ ** & $t(40)=5,12 ; p=0,001$ \\
\hline $\begin{array}{l}\text { H6 Est conscient de l'effet } \\
\text { des émotions et des } \\
\text { actions des élèves sur } \\
\text { lui-même }\end{array}$ & $3,41(0,50)$ & $-0,690$ ** & $t(40)=6,91 ; p=0,000$ \\
\hline
\end{tabular}

\section{Discussion et conclusion}

Les résultats de la présente étude confirment la première hypothèse en faisant ressortir des corrélations négatives et significatives entre les sous-échelles DIE et DDE de la TAS-20 et les 6 habiletés retenues dans la grille d'évaluation de stage se rapportant à des CSE. On se souviendra que l'échelle DIE mesure la difficulté à identifier ses émotions et l'échelle DDE évalue la difficulté à décrire ses émotions. Les analyses révèlent plus précisément une force d'association élevée $(r>-0,6)$ entre le fait d'avoir de la difficulté à identifier et à décrire ses émotions et 


\section{2}

les habiletés à tenir compte du côté affectif des élèves (habileté 2), à être facile d'approche et se rendre disponible pour les élèves (habileté 3 ) et faire preuve d'ouverture d'esprit et de flexibilité envers les élèves (habileté 4). De plus, la difficulté à décrire ses émotions est pour sa part spécifiquement et fortement associée au fait d'être conscient de l'effet des émotions et des actions des élèves sur soi-même et sur les élèves (habiletés 5 et 6). En d'autres termes, indépendamment du groupe auquel ils appartiennent, les stagiaires qui ont le plus de facilité à identifier et à décrire leurs émotions sont ceux qui rencontrent le moins de difficulté pour prendre en compte le côté affectif des élèves de leur classe, pour se rendre facile d'approche et disponible auprès d'eux et pour faire preuve d'ouverture d'esprit et de flexibilité. Dans le même sens, les stagiaires pour qui il est plus facile de décrire leurs émotions sont, quant à eux, plus à même d'être conscients des effets des émotions et des actions des élèves sur eux-mêmes et sur les élèves. Des constats similaires ont été rapportés dans l'étude de Perry, Lennie et Humphrey (2008) conduite auprès d'enseignants du primaire provenant du Nord-Ouest de l'Angleterre. Dans cette étude, les auteurs soulignent l'importance pour les enseignants de développer leurs CSE pour leurs propres bénéfices et celui de leurs élèves.

Relativement à la seconde hypothèse, les résultats de cette étude permettent de faire ressortir des niveaux d'acquisition variables des CSE entre les stagiaires alexithymiques et alexithymiques intermédiaires et les stagiaires non alexithymiques. Ces variations vont dans le sens de notre hypothèse puisque les scores des stagiaires alexithymiques et alexithymiques intermédiaires à l'échelle de la TSA-20 sont significativement plus bas à chacune des échelles de satisfaction des 6 habiletés associées à des CSE lorsqu'on les compare aux résultats des stagiaires non alexithymiques. Ces résultats corroborent ceux de Valesco, Fernández, Páez et Campos (2006) qui ont relevé un faible niveau de CSE chez des étudiants alexithymiques inscrits dans un programme de psychologie et provenant d'une université espagnole il y a plus de 10 ans. Ces résultats ne sont donc pas étonnants si l'on considère que les personnes alexithymiques ont de la difficulté à percevoir, exprimer, comprendre et réguler leurs émotions; des habiletés pourtant essentielles chez l'individu compétent sur le plan émotionnel (Mikolajczak et al., 2014).

De façon plus générale, ces résultats viennent renforcer nos préoccupations quant à la capacité des stagiaires alexithymiques et intermédiaires d'acquérir des CSE. Si tel est le cas, devrait-on se questionner davantage sur la façon dont les stagiaires en éducation développent leurs CSE? Serait-il judicieux d'offrir des ateliers sur le développement de CSE aux formateurs qui accompagnent les stagiaires en éducation? Pharand et Moreau (2017) s'intéressent à ces questions et mènent des recherches intéressantes dans le domaine. Ces auteurs ont recours au terme " littératie émotionnelle " lorsqu'ils font référence aux CSE et leurs travaux ont pour but d'outiller les enseignants à mieux réguler leurs émotions. En 2010 déjà, ces mêmes auteurs ont testé une approche prometteuse qui favorise l'autorégulation des émotions tant chez les enseignants que chez les élèves. II serait intéressant d'examiner si cette approche peut être utilisée dans le cadre de stages en éducation, voire intégrer dans un cours universitaire de gestion de classe ou d'éducation inclusive. 
Par ailleurs, cette étude présente un certain nombre de limites. D'abord, il est important de garder à l'esprit que l'outil retenu pour évaluer les CSE ne mesure que certaines d'entre elles, par exemple être conscient de l'effet de ses émotions et de ses actions sur les élèves, et a été construit à des fins de formation. Par conséquent, le profil des CSE des participants demeure partiel, car la grille d'évaluation utilisée pour les stages est conçue pour mesurer des habiletés liées à certaines CSE et non pour mesurer l'ensemble d'entre elles. Pour combler cette lacune, il serait avantageux d'ajouter un instrument standardisé tel que le Profil des Compétences Émotionnelles (PCE) mis au point par Brasseur, Grégoire, Bourdu et Mikolajczak (2013) ou le questionnaire de régulation émotionnelle (QRE) développé par Gross et John (2003). À l'aide de ces questionnaires, il serait envisageable de dresser un profil plus fidèle, plus précis et plus exhaustif des CSE des futurs enseignants. Ensuite, bien que l'environnement des stagiaires était relativement uniforme (écoles situées dans des quartiers de classes sociales similaires, écoles inclusives, etc.), d'autres variables ont pu avoir une incidence sur le niveau de performance des stagiaires. Nous faisons référence ici aux écarts entre les enseignants accompagnateurs et les professeurs conseillers quant à leurs CSE ou encore la sévérité des difficultés qu'éprouvent les élèves ayant des besoins éducatifs particuliers, notamment ceux ayant des troubles du comportement et pour lesquelles les CSE peuvent faire défaut. Enfin, la taille de l'échantillon constitué nous a seulement permis d'adopter un devis de recherche de nature descriptive corrélationnelle et par conséquent aucun lien de causalité entre les variables étudiées n'a pu être établi.

Outre les limites énoncées, cette étude comprend également certaines forces. Comme mentionné, il s'agit d'une étude novatrice qui vient enrichir les rares données qui existent sur l'alexithymie chez les futurs enseignants. Cette étude vient également renforcer l'idée qu'il est essentiel de s'assurer que les compétences visées dans tout programme de formation à l'enseignement construit sur la base d'une approche par compétences soient enseignées explicitement et évaluées de manière rigoureuse tout au long du cursus de l'étudiant. Ce constat est d'autant plus vrai que comme déjà mentionné les CSE ont été démontrées comme fondamentales dans l'exercice de la profession enseignante et que de nombreux chercheurs et professionnels de terrain s'entendent pour dire que les étudiants qui se destinent à l'enseignement devraient parfaire leurs CSE durant leur formation initiale (Cross et Hong, 2012; Lafortune, 2005; Visioli, Petiot et Ria, 2015). Finalement, dans l'éventualité de la création d'un cours de niveau universitaire sur les CSE, il nous semble primordial qu'un tel cours fasse l'objet de recherches empiriques afin de s'assurer qu'il réponde convenablement aux besoins particuliers des étudiants alexithymiques qui se destinent à l'enseignement ou à l'intervention psychosociale auprès d'enfants et d'adolescents présentant des problèmes de comportement. 


\section{Références}

Appleton, A. A., Loucks, E. B., Buka, S. L. et Kubzansky, L. D. (2014). Divergent associations of antecedent-and response-focused emotion regulation strategies with midlife cardiovascular disease risk. Annals of Behavioral Medicine, 48, 1-10. doi: 10.1007/ s12160-014-9600-4

Aucoin, A., Bélanger, N., Berg, L.C., Duchesne, H., Kropielnicki, B., Rousseau, N., Vienneau, R. (2013). Éducation inclusive au Canada : Analyse comparative. Trois-Rivières, Québec : Laboratoire international sur l'inclusion scolaire.

Bagby, R.M., Taylor G.J. et Parker J.D.A. (1994). The twenty-item Toronto alexithymia scale- II. Convergent, discriminant and concurrent validity. Journal of Psychosomatic Research, $38,33-40$.

Beckers, J. (2002). Développer et évaluer des compétences à l'école : vers plus d'efficacité et d'équité. Bruxelles, Belgique : Labor.

Boutin, G. (2004). L'approche par compétences en éducation : un amalgame paradigmatique. Connexion, 1, 25-41. doi: 10.3917/cnx.081.0025

Boutin, G. et Julien, L. (2000). L'obsession des compétences. Son impact sur l'éducation et la formation des enseignants. Montréal, Québec Éditions Nouvelles.

Brasseur, S. Grégoire, J. Bourdu R. et Mikolajczak, M. (2013). The Profile of Emotional Competence (PEC): Development and Validation of a SelfReported Measure that Fits Dimensions of Emotional Competence Theory. PLoS ONE, 8(5). doi: doi.org/10.1371/journal. pone.0062635.

Correa, M. et Gervais, C. (2008). Les stages en formation à l'enseignement. Pratiques et perspectives théoriques. Montréal, Québec : Presses de I'Université du Québec.
Cross, D. et Hong, J. (2012). An examination of teachers' emotions in the school context. Teaching and Teacher Education, 28, 957-967.

Coulet, J.-C. (2016). Compétence, compétences transversales et compétences clés : peut-on sortir de l'impasse? Éducation et socialisation, 41, 71-81.

Decelles, S. (2014). L'enseignant du XXIe siècle : une réalité actualisée. Apprendre et enseigner aujourd'hui, 4, 15-19.

Desbiens, J.F., Borges, C. et Spallanzani, C. (2013). J'ai mal à mon stage. Problèmes et enjeux de la formation pratique en enseignement. Montréal, Québec : Presses de I'Université du Québec.

Desseilles, M. et Mikolajczak, M. (2013). Mieux vivre avec ses émotions. Paris, France : Odile Jacob.

Donges, U.S., Kresting, A. et Suslow, T. (2014). Alexithymia and Perception of Emotional Information: A Rewiew of Experimental Psychological Finding. Universitas Psychological, 13, 745-756. doi.org/10.11144/Javeriana.UPSY13-2. apei

Evans, J.D. (1996). Straightforward Statistics for the Behavioral Sciences. Brooks, WI: Cole Publishing.

Fortin, M.-F. et Gagnon, J. (2010). Fondements et étapes du processus de recherche, $3^{e}$ édition. Méthodes quantitatives et qualitatives. Montréal, Québec : Chenelière Éducation.

Gatta, M., Facca, I., Colombo, E., Svanellini, L., Montagnese, S. et Schiff, S. (2014). Alexithymia, psychopathology and alcohol misuse in adolescence: A population based study on 3556 teenagers. Neuroscience \& Medicine, 5 , 60-71. doi: 10.4236/nm.2014.51009

Gaussel, M. (2018). À l'école des compétences sociales. Dossier de veille de l'IFÉ, 121, 1-28.

Goleman, D. (1995). Emotional Intelligence. Why it can matter more than IQ. Institute of education sciences, 24, 4950. 
Grahay, M. (2006). Dangers, incertitudes et incomplétude de la logique de la compétence en éducation. Revue française de pédagogie, 154, 97-110.

Gross, J. J. (2015). Emotion regulation: Current status and future prospects. Psychological Inquiry, 26, 1-26. doi: org/ 10.1080/1047840X.2014.940781

Gross, J.J. et John, O.P. (2003). Individual Differences in Two Emotion Regulation Processes: Implications for Affect, Relationships, and Well-Being. Journal of Personality and Social Psychology, 85, 348-362.

Kinman, G., Wray, S. et Strange, C. (2011). Emotional labour, burnout and job satisfaction in UK teachers: The role of workplace social support. Educational Psychology, 31, 843-856. doi: org/10.10 80/01443410.2011.608650

Kooiman, C.G., Spinhoven, P. et Trijsburg, RW. (2002). The assessment of Alexithymia, a critical review of the literature and a psychometric study of the Toronto Alexithymia Scale-20. Journal of Psychosomatic Research, 53, 1083-1090.

Kotsou, I. (2016). Intelligence émotionnelle et management. Comprendre et utiliser la force des émotions. Bruxelles, Belgique : de Boeck Supérieur.

Lafollie, D., Flenghi, D. et Dedieu, L. (2014). Perspectives pour l'éducation émotionnelle en milieu scolaire. Revue Éducation, Santé, Société, 1, 243-250.

Lafortune, L. (2005). Développement de la compétence émotionnelle? Dans L. Lafortune, M.-F. Daniel, P.-A. Doudin, F. Pons et O. Albanese (dir.), Pédagogie et psychologie des émotions : vers la compétence émotionnelle (p. 3559). Québec, Québec : Presses de I'Université du Québec.

Langevin, R. et Laurent, A. (2016). L'impact d'un cours universitaire sur les compétences émotionnelles chez un groupe d'étudiants alexithymiques inscrits dans un programme de formation initiale à l'enseignement. Nouveaux c@ hiers de la recherche en éducation, 19, 11-16. doi: 10.7202/1040665ar
Lasnier, F. (2014). Les compétences de l'apprentissage à l'évaluation. Montréal, Québec : Guérin Universitaire.

LeBoterf, G. (2008). Repenser la compétence. Pour dépasser les idées reçues : quinze propositions. Paris, France : Éditions d'Organisation.

Loas, G., Otmani, O., Fremaux, D., Lecercle, C., Duflot, M. et Delahousse, J. (1996). Étude de la validité externe, de la fidélité et de la détermination des notes seuils des échelles d'alexithymie de Toronto chez un groupe de malades alcooliques. Encéphale, 22, 35-40.

Luminet, 0. (2019). Alexthymie, l'émotion indéchiffrable. Sciences Humaines, 317, 40-41.

Luminet, O., Bagby, R.M. et Taylor, G.J. (2018). Alexithymia. Advance in research, theory and clinical practice. Cambridge, United Kingdom: Cambridge University Press.

Luminet, O. et Lenoir, V. (2006). Alexithymie parentale et capacités émotionnelles des enfants de 3 et 5 ans. Enfance, 58, 335-356. doi: 10.3917/enf.584.0335

Luminet, O., Vermeulen, N. et Grynberg, D. (2013). L'alexithymie. Comment le manque d'émotions peut affecter notre santé. Bruxelles, Belgique : De Boeck.

Mikolajczak, M. et Desseilles, M. (2012). Traité de régulation des émotions. Bruxelles, Belgique : De Boeck.

Mikolajczak, M., Quoidbach, J., Kotsou, I. et Nélis, D. (2014). Les compétences émotionnelles. Paris, France : Dunod.

Monchatre, S. (2009). Normalisation des compétences et rationalisation pédagogique. Le cas de la formation technique au Québec. Recherches en éducation, 7, 41-53.

Pelletier, M.A. (2015). La gestion des émotions face aux situations stressantes à l'école : les finissants stagiaires en éducation préscolaire et enseignement primaire se sentent-ils prêts? Éducation et francophonie, 2, 201-218. doi: org/10.7202/1034492ar 
Pharand, J. et Clément, N. (2010). Le langage des émotions : une démarche de l'intégration de l'intelligence émotionnelle. Dans $M$. Hébert et $L$. Lafontaine (dir.), Littératie et inclusion. Outils et pratiques pédagogiques ( $p$. 109-126). Québec, Québec: Presses de l'Université du Québec.

Pharand, J. et Moreau, A. C. (2017). La littéracie émotionnelle : lire et comprendre les émotions pour mieux vivre. Psychologie préventive, 50, 3038.

Perrenoud, P. (2011). Quand l'école prétend préparer à la vie... Développer des compétences ou enseigner d'autres savoirs? Paris, France : ESF éditeur.

Perry, L., Lennie, C. et Humphrey, N. (2008) Emotional literacy in the primary classroom: teacher perceptions and practices. Education 3-13, 1, 27-37. doi:10.1080/03004270701576851

Reicherts, M., Genoud, P.A. et Zimmermann, G. (2012). L'ouverture émotionnelle. Bruxelles, Belgique : Mardaga.

Romero, M. (2017). Enseignantes et enseignants, ces superhéros de l'éducation. Repéré à http://www. contact.ulaval.ca/article_blogue/ enseignantes-enseignants-superherosde-leducation/.
Salovey, P. et Mayer, J. D. (1990). Emotional intelligence. Imagination, Cognition and Personality, 9, 185-211.

Sander, D. (2015). Le monde des émotions. Paris, France : Belin.

Sander, D. et Scherer, K.R. (2014). Traité de psychologie des émotions. Paris, France : Dunod.

Sifneos PE. (1973). The prevalence of alexithymia characteristics in psychosomatic patients. Psychotherapy and Psychosomatics, 22, 255-262.

Tardif, J. (2006). L'évaluation des compétences. Montréal, Québec : Chenelière Éducation.

Tardif, M. (2013). La condition enseignante au Québec du XIXe au XXIe siècle. Une histoire cousue de fils rouges: précarité, injustice et déclin de l'école publique. Québec, Québec : Presses de I'Université Laval.

Valesco, C., Fernández, T., Páez, D. et Campos, M. (2006). Perceived emotional intelligence, alexithymia, coping and emotional regulation. Pscicothema, 18, 89-94.

Visioli, J., Petiot, O. et Ria, L. (2015). Vers une conception sociale des émotions des enseignants? Carrefour de l'éducation, 40, 201-230. doi: 10.3917/ cdle.040.0201 